\title{
Perceção das experiências académicas e saúde mental na adaptação ao ensino superior: que relações?
}

\author{
Carla Fonte \& Inês Macedo
}

\begin{abstract}
Resumo
O ensino superior e a adaptação académica requerem que os jovens se confrontem com múltiplas e complexas tarefas e as resolvam de uma forma mais ou menos bem sucedida. Esta nova fase da trajetória de vida apresenta-se como um desafio, podendo este impactar ao nível da saúde mental e do bem-estar dos estudantes. Neste âmbito apresenta-se um estudo cujo objetivo é caracterizar e analisar a relação entre os níveis de saúde mental/bem-estar e saúde mental/psicopatologia e a perceção das experiências académicas em estudantes universitários. Participaram 234 estudantes portugueses com idades entre os 17 e os 56, com uma média de 23.71 e desvio-padrão 5.508. Desses estudantes, 178 eram do sexo feminino e 56 do sexo masculino. Os dados foram recolhidos utilizando um Questionário sociodemográfico, a Escala Continuum de Saúde Mental-Versão Reduzida (Adultos) (MHC-SF), a Escala de Ansiedade, Depressão e Stress (EADS-21) e o Questionário de Vivências Académicas (QVA-r). Os resultados obtidos indicam que, globalmente, uma maior percepção e satisfação com a vida académica, se associa a melhores níveis de saúde mental e bem-estar, indicando que, os desafios enfrentados pelos estudantes, nos domínios pessoal, social, académico, institucional e vocacional, são variáveis relevantes para o seu processo de ajustamento, favorecendo assim os seus níveis de saúde mental e bem-estar.
\end{abstract}

Palavras-chave:

estudantes universitários; saúde mental; vivências académicas; adaptação ao ensino superior. 


\title{
Academic experiences perception and mental health in University education adaptation: what relationships?
}

\begin{abstract}
Higher education and academic adaptation require young people to confront themselves with multiple and complex tasks and to solve them in a more or less successful way. This new phase of life trajectory presents itself as a challenge, which may impact on the level of mental health and the well-being of the students. A study is presented with the objective to characterize and analyze the relationship between levels of mental health / well-being and mental health / psychopathology and the perception of academic experiences in university students. A total of 234 Portuguese students aged 17 to 56 participated, with a mean of 23.71 and a standard deviation of 5,508. Of these students, 178 were female and 56 were male. Data were collected using a Sociodemographic Questionnaire, the Mental Health Continuum - Short-Form (MHC-SF), the Depression, Anxiety and Stress Scale (DASS-21) and the Academic Experience Questionnaire. The results indicate that, overall, greater perception and satisfaction with academic life, is associated with better levels of mental health and well-being, indicating that the challenges faced by students in the personal, social, academic, institutional and vocational skills, are variables that are relevant to their adjustment process, thus favoring their levels of mental health and well-being.
\end{abstract}

Key-words: University students; Mental health; Academic Experiences; Adaptation to Higher Education.

\section{Perception des expériences universitaires et de la santé mentale lors de l'adaptation à l'enseignement supérieur: quelles relations?}

Resumé: L'enseignement supérieur et l'adaptation académique obligent les jeunes à se confronter à des tâches multiples et complexes et à les résoudre avec plus ou moins de succès. Cette nouvelle phase du parcours de vie se présente comme un défi pouvant avoir un impact sur le niveau de santé mentale et le bien-être des élèves. Cette étude présente une étude dont l'objectif est de caractériser et d'analyser la relation entre les niveaux de santé mentale / bien-être et de santé mentale / psychopathologie et la perception des expériences académiques chez les étudiants universitaires. Au total, 234 étudiants portugais âgés de 17 à 56 ans ont participé, avec une moyenne de 23,71 et un écart type de 5 508. Parmi ces étudiants, 178 étaient des filles et 56 des garçons. Les données ont été collectées à l'aide d'un questionnaire sociodémographique, du Continuum de la version à santé réduite (MHC-SF), de l'échelle d'anxiété, de dépression et de stress (EADS-21) et du questionnaire de l'expérience universitaire). Les résultats indiquent que, globalement, une perception et une satisfaction accrues vis-à-vis de la vie universitaire sont associées à de meilleurs niveaux de santé mentale et de bien-être, ce qui indique les compétences professionnelles sont des variables pertinentes pour leur processus d'adaptation, favorisant ainsi leur niveau de santé mentale et leur bien-être.

Mots-clés: Étudiants Universitaires; Santé Mentale; Expériences Académiques; Adaptation à L'enseignement Supérieur

\section{Percepción de las experiencias académicas y la salud mental en la adaptación a la educación superior: qué relaciones?}

Resumen: La Enseñanza Superior y la adaptación académica requieren que los jóvenes se enfrenta a múltiples y complejas tareas y las resuelvan de una forma más o menos exitosa. Esta nueva fase de la trayectoria de vida se presenta como un desafío, pudiendo este impacto en el nivel de la salud mental y del bienestar de los estudiantes. En este ámbito se presenta un estudio cuyo objetivo es caracterizar y analizar la relación entre los niveles de salud mental / bienestar y salud mental / psicopatología y la percepción de las experiencias académicas en estudiantes universitarios. Colaboradores 234 estudiantes portugueses entre las edades de 17 y 56 años, con una media de 23,71 y la desviación estándar de 5,508. De estos estudiantes, 178 eran del sexo femenino y 56 del sexo masculino. Los datos fueron recogidos utilizando un Cuestionario sociodemográfico, la Escala Continuum de Salud Mental-Versión Reducida (Adultos) (MHC-SF), la Escala de Ansiedad, Depresión y Stress (EADS-21) y el Cuestionario de Vivencias Académicas (QVA-r). Los resultados obtenidos indican que, globalmente, una mayor percepción y satisfacción con la vida académica, se asocia a mejores niveles de salud mental y bienestar, indicando que, los desafíos a los que se enfrentan los estudiantes, en los ámbitos personal, social, académico, institucional y institucional, vocacional, son variables relevantes para su proceso de ajuste, favoreciendo así sus niveles de salud mental y bienestar.

Palabras clave: Estudiantes universitarios; Salud mental; Vivencias académicas; Adaptación a la enseñanza superior. 


\section{Introdução}

O ingresso no ensino superior pode ser considerado como uma importante fase de transição para a vida adulta acarretando para os jovens estudantes um leque de novidades como por exemplo: o espaço institucional, normas particulares, tarefas académicas que exigem competências de estudo mais elevadas, maior autonomia e mais envolvimento por parte do aluno (Silveira, Norton, Brandão, \& Roma-Torres, 2011). Neste contexto os estudantes do ensino superior podem estar sujeitos a inúmeras pressões, desde a separação física dos pais, à exigência do ensino, à adaptação a uma nova etapa (amigos, novo meio físico, entre outros), bem como à necessidade de responder às expectativas que são depositadas sobre ele. Este momento de transição educativa, pode implicar a realização de novas tarefas que segundo Almeida, Soares e Ferreira (2000) as poderemos organizar em quatro domínios. O primeiro, o académico, refere-se à transição exigente que se verifica entre o Ensino Secundário e o Universitário, devido adaptações constantes aos novos ritmos e estratégias de aprendizagem, aos novos sistemas de ensino e de avaliação. O segundo, designado de social, salienta o facto de a entrada para a universidade acarretar o desenvolvimento de padrões de relacionamento interpessoal mais maduros na relação com a família, com os professores e os colegas, e com as figuras de autoridade. O terceiro, a nível mais pessoal, refere-se à dimensão que salienta a ideia de que nos anos do ensino superior devem concorrer para o estabelecimento de um forte sentido de identidade, para o desenvolvimento da auto-estima, de um maior conhecimento de si e para o desenvolvimento de uma visão pessoal do mundo. Por último, e em quarto lugar, o vocacional, já que o ensino superior constitui uma etapa importante no desenvolvimento da identidade vocacional, onde os processos de tomada de decisão, exploração e compromisso com objectivos merecem particular destaque (Almeida et al, 2002). Face a esta multiplicidade de aspetos a considerar na transição e na adaptação dos alunos, é fácil aceitar que o ajustamento ao contexto universitário se terá que assumir como um processo complexo e multidimensional. Isto porque todas estas novas tarefas desenvolvimentais podem impactar ao nível do ajustamento do próprio estudante, mais especificamente, em termos da sua saúde mental (Furegato, Santos \& Silva, 2010). Parece assim que os indivíduos inseridos na comunidade académica podem, por um lado, estar sujeitos a crises, podendo, por outro, estar num momento que pode ser aproveitado como uma oportunidade de crescimento (Silveira et al., 2011). Neste sentido, este momento e contexto de vida parece acarretar algum risco mas também oportunidades de desenvolvimento ao nível da saúde mental.

Ao longo da história existiram três diferentes conceções de saúde mental (Keyes, 2007). A primeira abordagem foi a patogénica, e a visão mais historicamente dominante, adveio da palavra grega pathos, que significa sofrimento ou uma simpatia 
que invoque emoção. Esta abordagem perceciona a saúde como a ausência de deficiência, doença e morte prematura (Keyes, 2007). A segunda conceção é a salutogénica que provém da palavra grega salus (saúde), que defende que a saúde implica a presença de estados positivos das capacidades humanas e o funcionamento do pensamento, da perceção e do comportamento (Keyes, 2007). O terceiro e último é o modelo do estado completo que deriva da palavra de saúde como sendo hale significando todo e forte - e esta abordagem é o exemplo da definição de saúde da Organização Mundial de Saúde (WHO, 2003), que defende a saúde como um estado completo de bem-estar, donde pertencem as capacidades positivas do indivíduo e o seu funcionamento, bem como a ausência de doença ou enfermidade (Keyes, 2007). Neste âmbito, medir a saúde mental pode ser então considerado um processo um tanto ou quanto difícil visto ter que se percecionar os parâmetros positivos na saúde do indivíduo, bem como os parâmetros que provocam sofrimento e mal-estar ao mesmo (Tengland, 2007). Segundo o Manual de Diagnóstico e Estatística dos Transtornos Mentais - $5^{a}$ Edição (DSM-V), a psicopatologia pode ser compreendida como uma síndrome caracterizada por uma perturbação clinicamente significativa na cognição de um indivíduo, na regulação da emoção ou comportamento que reflete uma disfunção nos processos psicológicos, biológicos ou de desenvolvimento subjacentes ao funcionamento mental (APA, 2013). No entanto, a ausência de psicopatologia não implica necessariamente que haja a presença de sentimentos positivos e do bom funcionamento do indivíduo tanto a nível pessoal como social. Deste modo a presença de psicopatologia per si não é um indicador sólido de que o indivíduo possa experienciar baixo bem-estar emocional, psicológico ou social (Lamers, Westerhof, Bohlmeijer, Klooster, \& Keyes, 2011). Atualmente, a saúde mental é descrita como sendo muito mais do que a ausência de psicopatologia (Lamers et al., 2011). Segundo a Organização Mundial de Saúde (WHO, 2003), a saúde mental pode ser considerada como um estado de bem-estar no qual o sujeito lida com o stress normal no seu dia-a-dia, trabalha produtivamente e consegue contribuir para a sociedade atual ou para a sua comunidade (WHO, 2003). Nesta definição podemos percecionar três conceitos diferentes: bem-estar, funcionamento pessoal positivo e funcionamento social positivo, que juntos demonstram a saúde mental num indivíduo. Keyes (2002, 2005, 2007), define saúde mental positiva como sendo um espetro de sintomas de hedonismo e funcionamento positivo percecionados pelo próprio indivíduo e as suas avaliações face tanto às suas vidas como ao seu funcionamento ao longo da vida. Em suma, o conceito de saúde mental evoluiu bastante ao longo dos anos, sendo que passou de uma visão só focada na psicopatologia para um leque mais aberto onde existem mais variáveis que possam influenciar o estado de saúde mental do indivíduo. A saúde mental deixou de ser a ausência de psicopatologia, acabando por se focar mais numa vertente positiva e englobando conceitos como bem-estar, 
bem-estar subjetivo, bem-estar social e bem-estar psicológico (Keyes, 2002; 2005; 2007; Galinha \& Pais-Ribeiro, 2005).

Alguns autores têm vindo a salientar que o início da vida adulta é um período marcado por bastantes mudanças psicossociais, como a transição do ensino secundário para o mundo de trabalho ou para o ensino superior. Estas transições estão talhadas por momentos desafiantes relacionados com o estabelecimento de novos vínculos, adaptação a um novo modelo de aprendizagem e avaliação, estabelecimento de identidade de carreira, entre outros (Almeida et al., 2000). Pode assim, ser um momento impactante ao nível da saúde mental do indivíduo, quer a nível psicopatológico, quer do seu bem-estar e funcionamento positivo. Parece assim pertinente aprofundar o estudo sobre o impacto que a qualidade da adaptação ao contexto universitário poderá ter sobre a sua saúde mental de jovens estudantes.

Neste âmbito desenvolveu-se um estudo cujo objetivo é caracterizar e analisar a relação entre os níveis de saúde mental/bem-estar e saúde mental/psicopatologia e a perceção das experiências académicas em estudantes universitários.

\section{Método}

\section{Participantes}

Este estudo contém uma amostra por conveniência, constituída por 234 estudantes universitários, onde 178 participantes são do sexo feminino e 56 do sexo masculino. As idades estão compreendidas entre os 17 e os 56 anos (média de 23.71 e o desvio-padrão de 5.508). Quanto ao estado civil, a maior parte dos inquiridos afirma ser solteiro (94.4\%), enquanto que 3\% diz ser casado, 0.9\% divorciado e $1.7 \%$ vive em união de facto. A maioria dos estudantes comtemplados neste estudo afirma estar integrado, no $4^{\circ}$ ano do ensino superior (26.5\%), em contraposição com os restantes que se inserem no $1^{\circ}$ ano (5.1\%), $2^{\circ}$ ano (17.9\%), $3^{\circ}$ ano (18.4\%), $5^{\circ}$ ano $(26.1 \%), 6^{\circ}$ ano (3.4\%), doutoramento (2.1\%) e pós-graduação (0.4\%). No que concerne à entrada no ensino superior implicar que os participantes abandonem o seu lar, 40.6\% respondem que sim, contrariamente aos restantes $58.5 \%$ que se mantiveram em suas casas. Os inquiridos que anteriormente referiram ter que mudarem de casa devido ao ingresso no ensino superior acabaram por viver num apartamento com outros estudantes $(0.9 \%)$, num apartamento sozinhos $(0.4 \%)$, num apartamento/casa com familiares (0.4\%), num apartamento com amigos (0.4\%), numa república de estudantes (5.1\%), numa casa com o(a) namorado(a) (3.8\%), num apartamento com trabalhadores (4.7\%) ou, como mostrou ser a maioria, num apartamento com outros estudantes (26.5\%). Por último, 169 inquiridos revelaram não exercer qualquer tipo de função associativa, contrariamente aos restantes 65 que se dividem por experiências associativas na universidade (72.2\%), experiências associativas fora da universidade/recreativas (11.1\%) e regime de voluntariado $(2.6 \%)$ 


\section{Instrumentos}

- Questionário sócio-demográfico

O questionário sócio-demográfico tem como objetivo recolher dados para descrever e caracterizar a amostra do presente estudo. Este foi composto por 10 questões que diziam respeito ao sexo, idade, estado civil, curso, ano de escolaridade, instituição superior onde se encontra matriculado, se o ensino superior implicou a saída de casa e se sim onde se encontrava a viver e, por último, se desempenha algum tipo de funções em alguma atividade associativa e se sim, qual e onde.

\section{- Escala Continuum de Saúde Mental - versão reduzida adultos (MHC-SF)}

A versão reduzida do MHC-SF foi construída como um instrumento para avaliar a saúde mental positiva (Keyes, 2002). Consiste em 14 itens que permitem avaliar o bem-estar emocional que corresponde a três itens, o bem-estar psicológico com seis itens e o bem-estar social com cinco itens. Este instrumento foi adaptado e validado para a população portuguesa por Fonte, Silva, Vilhena e Keyes (2019). Na amostra do presente estudo o instrumento apresenta boa consistência interna com os valores obtidos do Alfa de Cronbach de 0.88 dimensão bem-estar emocional, 0.85 na dimensão bem-estar social e 0.89 no bem-estar psicológico.

\section{- Escala de ansiedade, depressão e stress (EADS-21}

A Escala de Ansiedade, Depressão e Stress (EADS-21) foi adaptada para Portugal por José Luís Pais Ribeiro, Ana Honrado e Isabel Leal em 2004. Avalia a ansiedade, depressão e stress (Pais Ribeiro, Honrado \& Leal, 2004). Neste estudo foi usada a versão de 21 itens (Pais Ribeiro et al., 2004). Cada uma das escalas está assente em sete itens que incluem diferentes conceitos. E cada item consiste numa afirmação transporta para sintomas negativos, sendo então pedido ao participante para que ao responder aplique sempre «na semana passada». Os valores obtidos do Alfa de Cronbach no presente estudo são 0.90 na escala de depressão, 0.86 na escala de ansiedade e 0.88 na escala de stress.

\section{- Questionário Vivências Académicas (QVA-r)}

O Questionário de Vivências Académicas é um instrumento de auto-relato que avalia o modo como os estudantes percecionam as suas experiências académicas no ensino superior (Soares, Almeida \& Ferreira, 2006). Adaptado para versão portuguesa e brasileira, o QVA - versão integral é constituída por 170 itens distribuídos por 17 subescalas e a versão reduzida é constituído por 60 itens distribuídos por 5 dimensões: pessoal, interpessoal, carreira, estudo e institucional (Soares et al., 2006). A população alvo são os alunos do ensino superior e os itens do instrumento incluem conceitos como self; perceção de bem-estar; adaptação ao curso; perspetivas de carreira; 
competências de estudo; gestão do tempo; relacionamento com os pares; atividades extracurriculares; interesse pela instituição, entre outros (Soares et al., 2006). No presente estudo, a consistência interna do QVA-r na dimensão Pessoal é de 0.87, na dimensão interpessoal o índice é de 0.67 , na dimensão carreira é de 0.81 , na dimensão estudo é de 0.73 e a de dimensão institucional é de 0.61 .

\section{Procedimento}

Como primeiro passo a dar na realização deste estudo procedeu-se ao pedido de autorização aos autores dos instrumentos a utilizar. No que concerne à Escala Continuum de Saúde Mental - versão reduzida (MHC-SF) o pedido foi feito à Professora Doutora Carla Fonte, que disponibilizou os instrumentos para utilização. Em relação aos restantes instrumentos: Escala de Ansiedade, Depressão e Stress (EADS) e o Questionário de Vivências Académicas - R (QVA-r), encontravam-se publicados o que significa que não requerem qualquer tipo de autorização para o seu uso. Adicionalmente, foi criado um questionário sócio-demográfico que visava recolher dados inerentes ao estudo e que, mais tarde, fossem suporte para uma boa caracterização da amostra.

Depois de recolhidas as autorizações e os respetivos instrumentos, o projeto de investigação foi apreciado pela Comissão de Ética da Universidade onde o mesmo foi desenvolvido para que pudesse dar um parecer quanto ao mesmo. A resposta do parecer foi positiva, dando assim a possibilidade de começar o estudo empírico. Posteriormente, os instrumentos foram introduzidos para uma versão online recorrendo ao Google Docs, que cria um link online onde os participantes podem submeter as suas respostas de forma segura e anónima. Antes de mais, é importante ressalvar que tanto o consentimento informado como todos os esclarecimentos relativos ao estudo e futuros procedimentos estavam contempladas logo no início do questionário online e os participantes só poderiam passar para a resposta aos instrumentos assim que consentissem participar de livre e espontânea vontade no estudo, assinalando essa mesma vontade colocando uma sinalética num quadrado criado propriamente para o efeito. A partir do momento em que o estudo se encontra pronto para ser divulgado, contactamos todos os Gabinetes de Comunicação e Associações de Estudantes de Universidades do País, através de e-mail explicando o propósito do estudo, fornecendo o link e pedindo que o mesmo fosse divulgado pelo corpo estudantil. Também foram utilizadas as redes sociais como o Facebook para divulgar o estudo entre amigos e colegas. Uma das mais-valias da divulgação do estudo foi o efeito snowball que teve, assim que foram enviados e-mails e feitos contatos para colegas de curso e amigos que divulgaram o estudo entre o seu grupo de pares. O link referente ao estudo ficou disponível durante dois meses e depois da data estipulada foi desativado para se passar para próxima fase de trabalho: análise dos dados. É importante sublinhar 
que todas as respostas ficavam guardadas na conta pessoal de e-mail, de forma a resguardar os participantes e os resultados do estudo. No final foi feito o download do documento em versão Excel que foi passado e adaptado para o programa estatístico Statistical Package for the Social Sciences - versão 22 (SPSS) onde mais tarde foi feita a análise de dados. Optou-se pela utilização de testes paramétricos por estarem cumpridos os pressupostos para a aplicação dos mesmos.

\section{Resultados}

Caracterização da amostra em termos dos níveis saúde mental/bem-estar e saúde mental/psicopatologia e das suas vivências académicas

Em relação às suas dimensões da saúde mental positiva (Tabela 1), o bem-estar psicológico é a dimensão com valores médios mais elevados (média $=21.00$ ). Seguem-se os valores relativos ao bem-estar social (média $=12.92$ ) e ao bem-estar emocional (média $=10.61$ ).

Tabela 1. Caracterização níveis de bem-estar emocional, bem-estar psicológico e bem-estar social na amostra

\begin{tabular}{cccc}
\cline { 2 - 4 } & $\begin{array}{c}\text { Bem-estar } \\
\text { Emocional }\end{array}$ & $\begin{array}{c}\text { Bem-estar } \\
\text { Social }\end{array}$ & $\begin{array}{c}\text { Bem-estar } \\
\text { Psicológico }\end{array}$ \\
\cline { 2 - 4 } $\begin{array}{c}\text { Média } \\
\text { Desvio }\end{array}$ & 10.61 & 12.92 & 21.00 \\
padrão & 2.986 & 5.424 & 6.020 \\
Mínimo & 0 & 0 & 2 \\
Máximo & 15 & 24 & 30 \\
\hline
\end{tabular}

Relativamente à saúde mental a nível da psicopatologia (ver Tabela 2), verifica-se que é a escala referente ao stress onde se encontram valores médios mais elevados, (média=6.70), seguido dos valores da depressão (média=4.16), e por último o de ansiedade com (média=3.54).

Tabela 2. Caracterização dos níveis de depressão, ansiedade e stress na amostra

\begin{tabular}{cccc}
\cline { 2 - 4 } Média & Depressão & Ansiedade & Stress \\
\cline { 2 - 4 } DP & 4.16 & 3.54 & 6.70 \\
Mínimo & 4.340 & 2.54 & 4.463 \\
Máximo & 0 & 0 & 0 \\
\hline
\end{tabular}


Os valores do Questionário de Vivências Académicas - reduzido, no que concerne às suas dimensões, os valores mais altos encontram-se na dimensão carreira (média=46.62), interpessoal (média=42.85), estudo (média=41.91), obtendo valores mais baixos nas dimensões pessoal (média=34.17) e institucional (média=32.55) (cf. Tabela 3).

O QVA-r avalia a satisfação dos estudantes que se prendem com as vivências académicas e quanto maior a pontuação obtida através do somatório dos itens maior o nível de satisfação dos mesmos. Neste estudo, a média é de 194.77, sendo o máximo apresentado de 268 e o mínimo de 103. Na média, os estudantes encontram-se satisfeitos com as suas vivências académicas, tendo melhores resultados nas áreas da carreira, seguida das áreas: interpessoal e estudo.

Tabela 3. Caracterização dos valores relativos às vivências académicas da amostra

\begin{tabular}{cccccc}
\cline { 2 - 6 } Média & Pessoal & Interpessoal & Carreira & Estudo & Institucional \\
\cline { 2 - 6 } DP & 34.17 & 42.85 & 46.62 & 41.91 & 32.55 \\
Mínimo & 9.643 & 6.385 & 7.544 & 6.786 & 4.681 \\
Máximo & 16 & 22 & 19 & 23 & 15 \\
\hline
\end{tabular}

\section{Análise da relação entre saúde mental/psicopatologia e saúde mental/bem- estar e as vivências académicas}

Com o objetivo de caracterizar e relacionar as vivências académicas com as dimensões da saúde mental ao nível do bem-estar e da psicopatologia recorreu-se à Correlação de Pearson. Na Tabela 4 são apresentados os resultados obtidos segundo a comparação feita entre as variáveis analisadas. Verifica-se que uma maior perceção das vivências académicas pessoais está associada a maiores níveis de depressão, ansiedade e stress. Por outro lado, constatamos uma correlação negativa na perceção das vivências académicas pessoais dos estudantes universitários e os valores de saúde mental, bem-estar emocional, bem-estar social e bem-estar psicológico, indicando que quando a primeira aumenta as restantes diminuem. O domínio interpessoal encontra-se negativamente associado com as suas escalas de depressão, ansiedade e stress, logo os estudantes com uma maior perceção relativamente às vivências académicas ao nível interpessoal têm um maior índice de depressão, ansiedade e stress. Verifica-se ainda que maiores níveis na dimensão interpessoal se associam positivamente a maiores níveis de bem-estar emocional, bem-estar social e bem-estar psicológico. Para os valores da dimensão carreira verificam-se correlações significativas e negativas com os níveis de depressão, logo quanto menor é a perceção do estudante universitário relativamente à sua carreira maior é o seu índice de depressão. Por outro lado constata-se que uma perceção de carreira alta se associa positivamente aos 
níveis de bem-estar emocional, bem-estar psicológico e bem-estar social. No domínio relativo ao estudo, os estudantes com uma menor perceção neste domínio têm um índice mais elevado nas escalas de depressão, ansiedade e stress, pois estão significativamente e negativamente correlacionadas. Por outro lado, verificam-se associações significativas e positivas em relação às escalas de bem-estar, indicando que quanto maior a perceção do estudante em relação ao seu estudo maiores os seus níveis de bem-estar emocional, social e psicológico. Por último, em relação à dimensão institucional, constatam-se valores de correlação negativos nas escalas de depressão e ansiedade indicando que sempre que a perceção ao nível institucional é menor os valores de ansiedade e depressão serão maiores. Para além disso, sempre que os valores de perceção da vivência académica a nível institucional são maiores, aumentam também os valores de saúde mental e bem-estar dos estudantes universitários já que verificam correlações estatisticamente significativas entre estas dimensões.

Tabela 4. Relação entre saúde mental/psicopatologia e saúde-mental/bem-estar e vivências académicas

\begin{tabular}{|c|c|c|c|c|c|c|}
\hline & & Pessoal & Interpessoall & Carreira & Estudo & Institucional \\
\hline Depressão & $r$ & $\begin{array}{c}0.658 \\
\text { 水水 }\end{array}$ & $\begin{array}{c}-0.231 \\
\text { 水 }\end{array}$ & $\begin{array}{c}-0.216 \\
\text { 水水 }\end{array}$ & $\begin{array}{r}-0.241 \\
\text { 水 }\end{array}$ & $\begin{array}{c}-0.222 \\
\text { 水水 }\end{array}$ \\
\hline Ansiedade & $r$ & $\begin{array}{c}0.555 \\
\text { 水 }\end{array}$ & $\begin{array}{c}-0.180 \\
* *\end{array}$ & -0.090 & $\begin{array}{c}-0.152 \\
*\end{array}$ & $\begin{array}{c}-0.200 \\
* *\end{array}$ \\
\hline Stress & $r$ & $\begin{array}{c}0.612 \\
\text { 水 }\end{array}$ & $\begin{array}{c}-0.182 \\
\text { 水水 }\end{array}$ & -0.068 & $\begin{array}{c}-.131 \\
*\end{array}$ & -0.80 \\
\hline $\begin{array}{l}\text { Bem-estar } \\
\text { Emocional }\end{array}$ & $r$ & 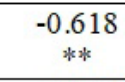 & $\begin{array}{c}0.390 \\
\text { 水水 }\end{array}$ & $\begin{array}{c}0.306 \\
\text { 水水 }\end{array}$ & $\begin{array}{c}0.333 \\
* * 2\end{array}$ & $\begin{array}{c}0.245 \\
\text { 水水 }\end{array}$ \\
\hline $\begin{array}{l}\text { Bem-estar } \\
\text { Social }\end{array}$ & $r$ & $\begin{array}{c}-0.489 \\
\text { 水 }\end{array}$ & $\begin{array}{c}0.466 \\
* *\end{array}$ & $\begin{array}{c}0.250 \\
\text { 水水 }\end{array}$ & $\begin{array}{c}0.380 \\
* *\end{array}$ & $\begin{array}{c}0.175 \\
* \cdots\end{array}$ \\
\hline $\begin{array}{l}\text { Bem-estar } \\
\text { Psicológico }\end{array}$ & $r$ & $\begin{array}{c}-0.564 \\
\text { 水水 }\end{array}$ & $\begin{array}{c}0.497 \\
\text { 水 }\end{array}$ & $\begin{array}{c}0.439 \\
\text { 水 }\end{array}$ & $\begin{array}{c}0.433 \\
\text { 水 }\end{array}$ & $\begin{array}{c}0.293 \\
\text { 水 }\end{array}$ \\
\hline
\end{tabular}

Nota. ${ }^{*}$ valores significativos $p<0.05^{* *}$ valores muito significativos $p<0.01$

\section{Discussão dos resultados}

O estudo apresentado teve como objetivo geral analisar a relação entre vivências académicas de estudantes universitários e os níveis de saúde mental/psicopatologia e saúde mental/bem-estar. Os resultados indicaram que os estudantes inquiridos apresentam globalmente níveis elevados de saúde mental positiva (bem-estar emocional, bem-estar psicológico e bem-estar social) e níveis baixos ao nível da psicopatologia (ansiedade, depressão e stress). Estes dados sugerem que a população em análise 
apresenta uma boa saúde mental, sendo os mesmos congruentes com outros estudos desenvolvidos junto de jovens portugueses (Fonte et al, 2019; Monte, Fonte \& Alves, 2015; Silveira et al., 2011).

Relativamente à perceção dos estudantes das suas vivências académicas os dados encontrados indicam que em termos gerais os estudantes mostram-se satisfeitos com a sua vivência académica, incidindo os valores mais altos nos domínios interpessoal, estudo e carreira. Estes resultados parecem refletir que, de um modo geral, o processo de transição e adaptação/ajustamento ao contexto universitário, sendo processo complexo e multidimensional e envolvendo múltiplos fatores tanto de natureza intra e interpessoal como de natureza contextual é percecionado pelos estudantes como sendo bem sucedido (Almeida et al, 2002). Importa neste aspeto ter em consideração que a forma como os indivíduos lidam com os momentos de transição e os resultados a que eles podem conduzir, poderá ser explicada quer pelas características pessoais do próprio indivíduo, quer as próprias características do contexto em si (Igue, Bariani \& Milanesi, 2008)

Da análise da relação entre saúde mental/psicopatologia e saúde mental/bem-estar e as vivências académicas foi possível constatar que uma maior percepção e satisfação com a sua vida académica se associam significativamente a melhores níveis de saúde mental e bem-estar sublinhando assim a importância deste momento e contexto na saúde mental dos indivíduos. Parece assim que a frequência do ensino superior, quando pautada por vivências académicas desafiadoras e exigentes, mas bem conseguidas, se apresentam como uma oportunidade de desenvolvimento positivo e de crescimento, podendo portanto ser um contexto de aquisição de novas competências, permitindo àqueles que por ele passam tornar-se indivíduos com melhor saúde mental, contribuindo assim para a sociedade atual ou para a sua comunidade (Almeida et al, 2000; Keyes, 2007).

Relativamente à dimensão mais pessoal verificamos que a mesma se associa positivamente a maiores níveis de psicopatologia (depressão, ansiedade e stress), e negativamente aos níveis de bem-estar (emocional, psicológico e social). Estes resultados podem ser explicados pelo fato destas variáveis mais pessoais, estarem relacionadas com o self e com o desenvolvimento psicossocial dos estudantes. Se quisermos, a par da adaptação às exigências académicas deste novo nível de ensino, o ingresso no Ensino Superior implica ainda, para um grande número de estudantes, o sair de casa, a separação dos amigos e das pessoas mais significativas, o confronto com um meio totalmente desconhecido, o decidir quem se é e o que se quer fazer da vida (Almeida et al, 2002). Questões como o estabelecimento de um forte sentido de identidade, o desenvolvimento de relações interpessoais mais maduras (com os pares, os professores e a família), a exploração de papéis sociais e sexuais, a resolução das questões da intimidade, assim como o estabelecimento de um plano de vida (incluindo a definição 
e o comprometimento com determinados objetivos pessoais e vocacionais), parecem assumir particular importância junto desta população. Desta forma, as questões da competência, da autonomia e da identidade assumem particular importância durante os anos de frequência universitária, o que poderá impactar na sua saúde mental, quer em termos do aumento da probabilidade de desenvolver psicopatologia, quer na diminuição do seu bem-estar (Igue, Bariani \& Milanesi, 2008).

Já na dimensão interpessoal os dados indicaram que à medida que os valores desta dimensão aumentam, aumentam também os níveis de bem-estar (emocional, psicológico e social) e diminuem os níveis de psicopatologia (depressão, ansiedade e stress). Estes dados estão em acordo com a perspetiva de alguns autores que têm salientado que, a experiência universitária, pode conduzir os estudantes a realizarem um aumento da qualidade das relações interpessoais, ao desenvolvimento de um forte sentido de identidade e ao aumento da auto-confiança (Almeida, et al. 2002).

Quanto à dimensão carreira, os resultados encontrados mostraram que menor perceção por parte dos estudantes nesta dimensão se associa a maiores níveis de depressão, já uma maior perceção na dimensão carreira se associa a maiores níveis de saúde mental positiva, concretamente bem-estar emocional, psicológico e social. Curiosamente também a dimensão estudo apresenta correlação negativa com a dimensão da saúde mental a nível da psicopatologia, indicando os resultados que menores perceções na dimensão estudo de associam a maiores níveis de depressão, ansiedade e stress. Estas dimensões das vivências académicas referem-se a aspetos da aprendizagem, como as bases de conhecimentos para o curso; métodos de estudo, por exemplo o acompanhamento das aulas, a consulta da bibliografia, a organização dos apontamentos; a realização de avaliações; o diálogo com os professores e a possibilidade de contactos dentro e fora das aulas (Almeida et al, 2000). Os resultados encontrados parecem indicar que, de algum modo, estas variáveis quando funcionam bem na perceção do próprio estudante poderão impactar positivamente na sua saúde mental, quer no aumento dos níveis de bem-estar quer na diminuição da psicopatologia. Evidentemente que deveremos também atender que o impacto destas variáveis pode também ser condicionado por outras como por exemplo o ano curricular em que o estudante se encontra.

Por último na dimensão institucional os resultados indicam que valores mais elevados na forma como os estudantes percecionam esta vivência académica indicam também maiores níveis de bem-estar emocional, psicológico e social e menores níveis de depressão e ansiedade. Estes dados dão um contributo na compreensão do papel da própria instituição na aprendizagem, adaptação e desenvolvimento psicossocial dos jovens universitários. Alguns autores têm vindo a salientar que a adequação dos settings, ou seja, dos espaços físicos (salas de aula, serviços, espaços de lazer, arquitetura, arranjos e limpeza, etc.) podem afetar o comportamento dos alunos e o seu 
rendimento académico (Gifford, 1997). Aqui, os sentimentos de espaços vivenciados pelos estudantes, associados a perceções de segurança, conforto e pertença, aparecem como variáveis igualmente importantes para descrever a adaptação e a realização académica dos estudantes (Almeida et al., 2002). Estas variáveis do contexto acabam por facilitar ou comprometer os processos adaptativos dos alunos, ou outros recursos disponibilizados para esse efeito, desempenhando um papel decisivo na persistência dos mesmos no curso e no seu envolvimento académico em geral, dados de alguma forma confirmados com os nossos resultados, pelas associações encontradas com os níveis de saúde mental.

\section{Conclusão}

Em suma, assumindo-se o papel central do estudante no processo de ensino-aprendizagem, parece relevante que todos os intervenientes no Ensino Superior reflitam como se organiza o ensino e os demais espaços de formação-aprendizagem, analisando também o grau de autonomia e de auto-regulação dos estudantes nas suas aprendizagens. O nosso estudo dá indicação de que uma melhor adaptação e vivência académica de associa a uma melhor saúde mental. Neste sentido, as instituições de Ensino Superior deverão estar atentas aos métodos de estudo implementados, à gestão do tempo e os comportamentos face à avaliação, quer de estudantes quer em professores. A par da sua qualificação científica, interessa atender competências pedagógicas e relacionais dos docentes do ensino superior. Estas competências são importantes, quer na adaptação ao curso quer, e sobretudo, no sucesso académico dos estudantes, pois que se refletem nos métodos de ensino e de avaliação. Almeida e colaboradores (2002) ressaltam que as instituições deverão proporcionar aos seus estudantes as condições adequadas para que experimentem o satisfatório conforto acadêmico, importante indicador da qualidade institucional e essencial para a qualidade da aprendizagem. Torna-se relevante promover a qualidade da vivência académica que engloba tanto uma nova filosofia curricular dos cursos e a organização das universidades, como a participação dos estudantes e das suas organizações estudantis. A universidade não se pode alhear das dificuldades vividas pelos jovens nesta sua transição. Este estudo procurou perceber como é que a perceção das vivências académicas por parte dos estudantes se associa à saúde mental dos mesmos. Em investigações futuras seria interessante adicionar mais variáveis de análise como por exemplo análise diferencial em termos de saúde mental de acordo com o ano frequentado pelos alunos; o tipo de recursos e apoios oferecidos pela instituição de ensino e ainda as diferentes áreas de formação. 


\section{Referências Bibliográficas}

Almeida, L. S., Soares, A. P. \& Ferreira, J. (2000). Transição e adaptação à universidade Apresentação de um Questionário de Vivências Académicas (QVA). Psicologia, X IV (2), 189 -208.

Almeida, L. S., Soares, A. P. C., Vasconcelos, R. M., Capela, J. V., Vasconcelos, J. B., Corais, J. M. \& Fernandes, A. (2002). Envolvimento extracurricular e ajustamento académico: Um estudo sobre as vivências dos estudantes universitário com e sem funções associativas. In A. P. Soares, A. Osório, J. V. Capela, L. S. Almeida, R. M. Vasconcelos \& S. M. Caires (Eds.), Transição para o Ensino Superior (167-187). Braga: Universidade de Minho, Conselho Académico.

American Psychiatric Association. (2013). Diagnostic and statistical manual of mental disorders. (5thEd.). London: British Library.

Fonte, C., Silva, I., Vilhena, E. et Keyes, C. (2019). The Portuguese Adaptation of the Mental Health Continuum-Short Form for Adult Population. Community Mental Health Journal, 1-8. https://doi. org/10.1007/s10597-019-00484-8.

Furegato, A. R. F., Santos, J. L. F. \& Silva, E. C. (2010). Depressão entre estudantes de dois cursos de enfermagem: auto-avaliação da saúde e fatores associados. Revista Brasileira de Enfermagem, 63(4), 509-516.

Galinha, I. \& Pais Ribeiro, J. L. (2005). História e evolução do conceito de bem-estar subjectivo. Psicologia, saúde e doenças, 6(2), 203-214.

Gifford, R. (1997). Environmental psychology: Principles and practice. Needham Heights: Allyn \& Bacon.

Igue, E:, Bariani, I. \& Milanesi, P. (2008). Vivência acadêmica e expectativas de universitários ingressantes e concluintes, Psico-USF, 13 (2), 155-164.

Keyes, C. L. M. (2002). The mental health continuum: from languishing to flourishing in life. Journal of Health and Social Research, 43, 207-222.

Keyes, C. L. M. (2005). Mental illness and/or mental health? Investigating axioms of the complete state model of health. Journal of Consulting and Clinical Psychology, 73(3), 539-548. doi: 10.1037/0022006X.73.3.539

Keyes, C. L. M. (2007). Promoting and protecting mental health as flourishing - a complementary strategy for improving national mental health. American Psychologist, 62(2), 95-108. doi: 10.1037/0003066X.62.2.95

Lamers, S. M. A., Westerhof, G. J., Bohlmeijer, E., Klooster, P., \& Keyes, C. (2011). Evaluating the psychometric properties of the mental health continuum-short form (MHC-SF). Journal of Clinical Psychology, 67, 99-110. doi: 10.1002/jcl p.20741

Monte, K., Fonte, C. \& Alves, S. (2015). Saúde mental numa população não clínica de jovens adultos: da psicopatologia ao bem-estar. Revista Portuguesa de Saúde Mental (Ed. Esp. 2): 83-87.

Neves, M. C. C. \& Dalgalarrondo, P. (2007). Transtorno mentais auto-referidos em estudantes universitários. Jornal Brasileiro de Psiquiatria, 56(4), 237-244.

Pais-Ribeiro, J., Honrado, A., \& Leal, I. (2004). Contribuição para o estudo da adaptação portuguesa das escalas de ansiedade, depressão e stress (EADS) de 21 itens de Lovibond e Lovibond. Psicologia, Saúde e Doenças, 5(2), 229- 239. 
Shin, K. \& You, S. (2013). Leisure type, leisure satisfaction and adolescents' psychological wellbeing. Journal of Pacific RIM Psychology, 2(7), 53-62.

Silveira, C., Norton, A., Brandão, I., Roma-Torres, A. (2011). Saúde mental em estudantes do ensino superior - experiência da consulta de psiquiatria do Centro Hospitalar São João. Acta Med Porto, 24(S2), 247-256.

Sequeira, M. M. M. \& Padovam, V. A. R. (2008). Bases teóricas de bem-estar subjectivo, bem-estar psicológico e bem-estar no trabalho. Psicologia: Teoria e Pesquisa, 24(2), 201-209.

Soares, A. P., Almeida, L. S., Ferreira, J. A. (2006). Questionário de Vivências Académicas: Versão integral (QVA) e versão reduzida (QVA-r). In: Gonçalvez, M. M., Simões, M. R., Almeida, L. S. Machado, C. Avaliação psicológica: Instrumentos validados para a população portuguesa, Coimbra: Quarteto, 1, 102-120.

Tengland, P. (2007). A two-dimensional theory of health. Theoretical Medicine and Bioethics, 28, $257-$ 285.

World Health Organization. (2003). Investing in mental health. Geneva, Switzerland: Department of Mental Health and Substance Dependence, Nuncommunicable Diseases and Mental Health.

\section{Carla Fonte}

Faculdade de Ciências Humanas e Sociais Universidade Fernando Pessoa

E-mail: cfonte@ufp.edu.pt ORCID: 0000-0002-7280-2083

Inês Macedo Faculdade de Ciências Humanas e Sociais Universidade Fernando Pessoa E-mail: inesmacedo@ufp.edu.pt

Correspondência:

Carla Alexandra Fonte- PhD

Praça 9 de Abril, $n^{\circ} 349$ 4249-004 Porto, Portugal

Data de submissão: Setembro de 2019 Data de avaliação: Dezembro de 2019 Data de publicação: Novembro 2020 